\title{
Relationship between Epstein-Barr Virus- Encoded RNA Expression, Apoptosis and Lymphocytic Infiltration in Gastric Carcinoma with Lymphoid-Rich Stroma
}

\author{
Mee-Yon Cho ${ }^{\mathrm{a}}$ Tae Heon Kim ${ }^{\mathrm{a}}$ Sang Yeop Yi ${ }^{\mathrm{b}}$ Woo Hee Jung ${ }^{\mathrm{c}}$ \\ Kwang Hwa Park ${ }^{\mathrm{a}}$ \\ Departments of Pathology, ${ }^{a}$ Wonju College of Medicine, Yonsei University, Wonju, ${ }^{b}$ College of Medicine, \\ Kwandong University, Goyang and ${ }^{\mathrm{C}}$ College of Medicine, Yonsei University, Seoul, Korea
}

\section{Key Words}

Carcinoma $\cdot$ Stomach $\cdot$ Gastric carcinoma with

lymphoid-rich stroma - Epstein-Barr virus · Apoptosis . bcl-2 $\cdot$ bax

\begin{abstract}
Objective: To investigate the association of apoptosis with apoptotic proteins and Epstein-Barr virus (EBV) expression in gastric carcinoma with lymphoid-rich stroma (GCLRS) in order to clarify the relationship between lymphocytic infiltration, EBV expression and apoptosis in gastric carcinoma. Subjects and Methods: We performed immunohistochemical stains for bcl-2, bax and in situ hybridization for EBV-encoded RNA and the terminal deoxynucleotidyl transferase-mediated dUTP-digoxigenin nick end labeling technique using the 24 cases of GCLRS and 23 cases of gastric carcinoma with lymphoid-poor stroma (GCLPS) out of 1,635 cases of gastric carcinoma. Results: The prevalence of GCLRS was $1.47 \%$ $(24 / 1,635)$. The prevalence of EBV involvement in GCLRS $(79 \%, 19 / 24)$ was significantly higher than in GCLPS $(8.7 \%, 2 / 23)$. Apoptosis was revealed in all cases and there was no statistical difference between GCLRS and GCLPS. Immunohistochemical expression for bcl-2 was
\end{abstract}

revealed only in the cytoplasm of lymphocytes in all cases, but bax showed distinct expression in the cytoplasm of neoplastic cells in all cases. There was a significant correlation between bax expression and the apoptotic index $(p<0.05)$. Lymphocytic infiltration was significantly correlated with the expression of EBV $(p<0.05)$ but not with survival rate and apoptosis. Most of the GCLRS cases were rated as low clinical stage (stage IA or B) and seemed to have a good prognosis. Conclusions: These results support the hypothesis that EBV infection plays an important role in the tumorigenesis of GCLRS, which has a better prognosis than usual gastric adenocarcinoma although there was no significant difference in the apoptotic index between GCLRS and GCLPS.

Copyright (c) 2004 S. Karger AG, Basel

\section{Introduction}

Gastric carcinoma is one of the cancers having a high mortality rate in Korea [1]. Although it is adenocarcinoma in most cases, gastric carcinoma with lymphoid rich stroma (GCLRS) can be seen in $1-4 \%$ of the patients [2-7]. The GCLRS is accompanied by definite lymphocytic infiltration around malignant cells, and therefore is

\section{KARGER}

Fax +4161306 1234 E-Mailkarger@karger.ch www.karger.com
C 2004 S. Karger AG, Basel $1011-7571 / 04 / 0136-0353 \$ 21.00 / 0$

Accessible online at: www.karger.com/mpp
Kwang Hwa Park

Department of Pathology, Wonju College of Medicine, Yonsei University

162 Ilsan-dong

Wonju, Kangwon-Do, 220-701 (South Korea)

Tel. +82 33741 1552, Fax +82 33731 6590, E-Mail abba@wonju.yonsei.ac.kr 
histologically differentiated from usual gastric adenocarcinoma. Studies have been conducted with this carcinoma in association with Epstein-Barr virus (EBV) due to its histologic similarity with nasopharyngeal lymphoepithelioma [5-9], which is a well-known EBV-associated tumor. Generally, EBV is expressed in $6.9-16 \%$ in gastric carcinoma $[10,11]$, whereas in association with lymphoid stroma its high value of $80 \%$ suggests the possibility that GCLRS could be a different cancer type compared with usual gastric carcinoma and its tumorigenesis.

Kume et al. [12] reported the expression of bcl-2 in EBV-positive gastric carcinoma and low apoptosis, suggesting a relationship between EBV and apoptosis. Unlike necrosis, apoptosis is active and programmed cell death and many studies have been conducted on apoptosis in carcinogenesis. A recent study reported that the resistance of EBV-encoded RNAs (EBERs) to apoptosis (via inhibition of the activity of double-stranded RNA-activated protein kinase) is important in the tumorigenesis of EBV-infected cells [13]. It is known that EBV latent membrane protein-1 (LMP-1) inhibits apoptosis by infecting not only B lymphocytes but also epithelial cells participating in carcinogenesis [14], suggesting that EBV could play a crucial role in apoptosis during oncogenesis of EBV-associated gastric neoplasia. Generally, no relationship was observed between apoptosis and apoptosis regulatory proteins in gastric adenocarcinoma [15]. Nonetheless, EBV expression was high in GCLRS compared to other types of gastric adenocarcinoma; thus, EBV is believed to play an important role in carcinogenesis and a possible relationship exists between EBV expression and apoptosis. It is known that bcl-2-induced EBV LMP-1 suppresses apoptosis and that bax, a gene with similar genetic structure to bcl-2, functions competitively in the cell with bcl-2 to form a heterodimer, and promotes apoptosis by functioning opposite to bcl-2 [16-18].

Thus, we reviewed surgically removed gastric carcinoma to determine the frequency of GCLRS and examined the relationship with EBV and changes in apoptosis, and apoptosis regulatory protein expressions, compared with gastric adenocarcinoma in general.

\section{Subjects and Methods}

\section{Subjects}

By reviewing 1,635 slides from patients who were diagnosed with gastric carcinoma and who underwent surgical resection from 1996 to 1999 at Wonju Christian Hospital, Wonju College of Medicine and Youngdong Severance Hospital, Yonsei University, we determined the incidence of GCLRS. The same number of gastric cancers with no lymphoid stroma was chosen by systematically sampling every 4 gastric cancer specimens among all gastrectomies that were registered in our department for 1 year. These served as the control group.

\section{Clinical Data}

The age, gender and tumor site were obtained from the medical records while the clinical stage of the gastric carcinoma (according to the criteria set by the American Joint Committee on Cancer [1]) was obtained from the pathologic reports.

\section{Histopathologic Tests}

Paraffin-embedded 5 - $\mu \mathrm{m}$-thick tissue sections were stained with hematoxylin-eosin and observed under the light microscope to confirm the histological type and the degree of lymphocytic infiltration. GCLRS was present when there was no proliferation of connective tissue in tumor stroma and lymphocytic infiltration was more than $30 \%$ of the tumor area (fig. 1). Those cases that did not fall into the GCLRS category were defined as gastric carcinoma with lymphoid-poor stroma (GCLPS).

\section{Immunohistochemical Staining}

bcl-2 primary antibody (DAKO, Denmark) used for immunohistochemical staining was diluted at 1:50. Immunohistochemical staining for bcl-2 was done using selected paraffin-embedded blocks cut into $5-\mu \mathrm{m}$ slices, which were placed on poly- $L$-lysine-coated slides and dried for $2 \mathrm{~h}$ at $50{ }^{\circ} \mathrm{C}$. After deparaffinization and rehydration, each slide was heated in a microwave oven $(750 \mathrm{~W})$ for $5 \mathrm{~min} 3$ times and kept in a pressure cooker for $20 \mathrm{~min}$. It was then treated with $3 \%$ hydrogen peroxide/methanol for $10 \mathrm{~min}$ for the inhibition of endogenous peroxidase. The slide was washed with distilled water and Tris buffer for $10 \mathrm{~min}$. It was reacted with the primary antibody solution overnight at room temperature and was washed with Tris buffer for $10 \mathrm{~min}$. Color development was done in 3-amino-9-ethylcarbazole (1:50 dilution) for $6 \mathrm{~min}$. The slide was then washed under running water, counterstained with hematoxylin, washed under running water and sealed with glycerol. bax primary antibody (Carpinteria, Calif., USA) for immunohistochemical staining was diluted at 1:100. The same treatment was done for bax as in the case of bcl-2 but the slides were maintained in an amplification reagent for 15 min using a Catalysed Signal Amplification kit (DAKO, Calif., USA) instead of microwaving, treated with streptavidin-peroxidase for $15 \mathrm{~min}$, and counterstained with hematoxylin. The slide was determined positive when malignant cells under the light microscope showed strong dark brown staining in their nuclei or cytoplasm. Furthermore, the degree of positivity of malignant cells was rated as $0,1+$, and $2+$ when the area showing positive expression was less than $10 \%$, between 10 and $30 \%$, and more than $30 \%$ of the total area inspected, respectively.

Staining of Apoptotic Cells Using Terminal DeoxynucleotidylMediated dUTP-Nick End Labeling (TUNEL)

ApopTag in situ apoptosis detection kit (Intergen, N.Y., USA) was used to examine apoptosis and staining was done as follows. After paraffin blocks were cut into $5-\mu \mathrm{m}$-thick slices and dried at room temperature, each slice was deparaffinized, rehydrated and treated with $20 \mu \mathrm{g} / \mathrm{ml}$ proteinase $\mathrm{K}$ for $25 \mathrm{~min}$ at room temperature. To block endogenous peroxidase, it was treated with 3\% peroxide solution containing phosphate-buffered saline. Using terminal de- 
Fig. 1. A Histological findings of GCLRS show dense lymphocytic infiltration with lymphoid follicle formation between the infiltrating adenocarcinoma cells. $\times 20$. B Higher magnification of $\mathbf{A}$ shows the adenocarcinoma to be composed of nests of poorly differentiated malignant epithelial cells containing large nuclei (white arrow). $\times 200$.
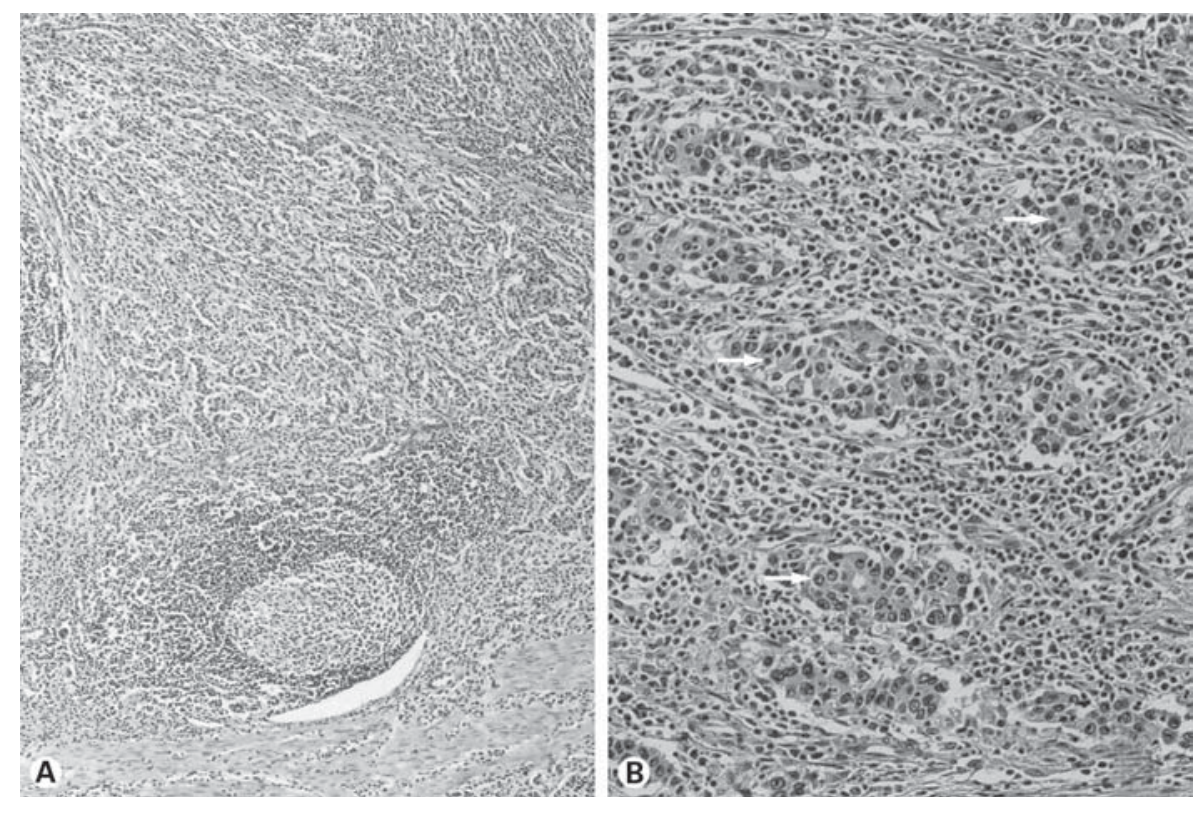

oxynucleotidyl transferase, the digoxigenin-labeled nucleotide was added to the $3^{\prime}-\mathrm{OH}$ end of fragmented DNA and antidigoxigeninperoxidase solution was placed on the slide. For color development, the slide was treated with diaminobenzidine hydrogen peroxidase and counterstained lightly with $0.5 \%$ methyl green. The apoptotic index was divided into $0,1+$, and $2+$ when the area of tumor showing positive expression was less than $10 \%$, between 10 and $30 \%$, and more than $30 \%$, respectively, by observing the histological site of well-stained nuclei under a low-power field. Under $\times 400$ magnification, 1,000 malignant cells per slide were counted to determine the percentage of positive cells and the data were used to determine the apoptotic index.

\section{In situ Hybridization for EBER}

After paraffin blocks were cut into 5 - $\mu$ m-slices and dried at $50{ }^{\circ} \mathrm{C}$, each slide was deparaffinized, rehydrated, reacted in $10 \mu \mathrm{g} / \mathrm{ml}$ proteinase $\mathrm{K}$ for $20-30 \mathrm{~min}$, and washed with distilled water. After dehydrating in serial concentrations of 95 and $99 \%$ ethanol, it was dried in air. For EBER 1-2 drops of oligonucleotide probe (Novocastra, Newcastle, UK) were placed on the slide, each slice was covered with a coverslip and reacted for $1 \mathrm{~h}$ in a water bath at $37^{\circ} \mathrm{C}$. It was washed with Tris buffer containing $0.1 \%$ Triton $\mathrm{X}-100$. For the prevention of nonspecific binding, it was reacted in anti-FITC/alkaline phosphatase reagent diluted 100-fold with $0.05 \mathrm{M}$ of Tris buffer for $30 \mathrm{~min}$. After washing with Tris buffer, it was reacted in alkaline substrate buffer solution for $5 \mathrm{~min}$ to allow for chromogens to penetrate into tissue. The slide was reacted in levamisole $(1 \mu \mathrm{l})$, added for the suppression of the chromogens nitroblue tetrazolium/5-bromo-4-chloro-3-indolylphosphate and endogenous alkaline substrate, diluted with alkaline substrate buffer at 50 -fold for $1 \mathrm{~h}$ and washed with Tris buffer. Counterstaining was done using hematoxylin. The sample was rated positive when more than $10 \%$ of malignant cells were strongly stained brown under the light microscope.

EBV-Encoded RNAs and Apoptosis in Gastric Carcinoma

\section{Statistical Analysis}

According to the results of determining the degree of lymphocytic infiltration and tumor site, and immunohistochemical staining for bcl-2 and bax, the relationship between EBV expression and clinical stage was investigated using $\chi^{2}$ test using SPSS 9.0 statistical software (Systat, USA). Student's t test was used to determine statistical significance in the relationship between the apoptotic index and the above variables. Furthermore, the Kaplan-Meier method was used to analyze the relationship between survival, the degree of lymphocytic infiltration, tumor site, and EBV expression in those cases that could be followed up; $p$ values less than 0.05 were considered to be statistically significant.

\section{Results}

The clinical and pathological characteristics of GCLRS and GCLPS are given in table 1 . Of the 1,635 cases of gastric carcinoma, GCLRS was seen in 24 cases, showing an incidence of $1.47 \%$. The gender ratios in patients with GCLRS and those with GCLPS were similar at 2:1 and 2.4:1, respectively, and no difference was seen in the average age at 55 and 56 years, respectively. Lesion sites in the cases of GCLRS were the body and cardia in 54\% of the patients and antrum in $46 \%$, showing slight predilection for the body and cardia. In GCLRS, the most prevalent clinical stages were stages IA and IB at $50 \%$, followed by stage II at $4.2 \%$, III at $45.8 \%$ and IV at $0 \%$. In GCLPS, on the other hand, the prevalence of stage I was $33.1 \%$, II $19.7 \%$, III $38 \%$, and IV 9.2\%, showing lower stages of GCLRS at the time of surgery. 
Fig. 2. In situ hybridization for EBER-1 shows strong positivity (white arrow) in nuclei of the malignant cells of a GCLRS sample. $\times 200$.

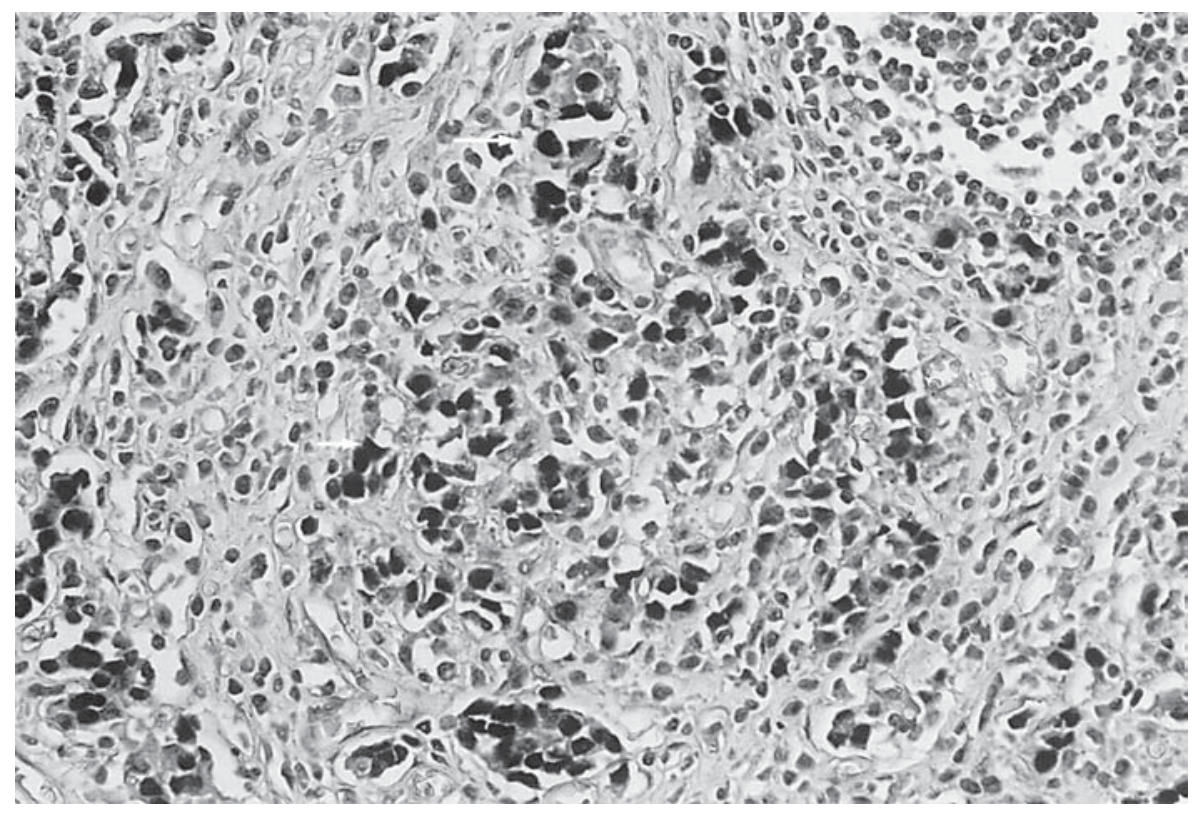

Table 1. Clinical characteristics of GCLRS and GCLPS

\begin{tabular}{llcc}
\hline & & GCLRS & GCLPS \\
\hline Sex & male:female & $2: 1$ & $2.4: 1$ \\
\hline Age, years & median age & 55 & 56 \\
\hline Site, $\%$ & antrum & 54 & 62 \\
& body and cardia & 46 & 38 \\
\hline Stage, $\%$ & IA & 16.7 & 22.2 \\
& IB & 33.3 & 10.9 \\
& II & 4.2 & 19.7 \\
& IIIA & 25.0 & 21.9 \\
& IIIB & 20.8 & 16.1 \\
& VI & 0.0 & 9.2 \\
\hline
\end{tabular}

Table 2. Relationship between lymphocytic infiltration and bax, apoptosis and EBER expression

\begin{tabular}{|c|c|c|c|c|}
\hline & & GCLRS $(n=24)$ & GCLPS $(n=23)$ & $\mathrm{p}$ value \\
\hline \multirow[t]{2}{*}{ bax } & $1+$ & 7 & 7 & \multirow[t]{2}{*}{ NS } \\
\hline & $2+$ & 17 & 16 & \\
\hline \multirow[t]{2}{*}{ Apoptosis } & $1+$ & 11 & 14 & \multirow[t]{2}{*}{ NS } \\
\hline & $2+$ & 13 & 9 & \\
\hline \multirow[t]{2}{*}{ EBV } & positive & 19 & 2 & \multirow[t]{2}{*}{$<0.001$} \\
\hline & negative & 5 & 21 & \\
\hline
\end{tabular}

\section{In situ Hybridization for EBER}

EBER expression was observed only in malignant cells but not in infiltrated lymphocytes around tumors and normal gastric mucosa (fig. 2). It was detected in 19 out of 24 cases of GCLRS, and in 2 out of 23 cases of GCLPS, the rates of EBER expression in GCLRS and GCLPS being 79 and 8.7\%, respectively; EBER expression in GCLRS was found to be statistically significant $(\mathrm{p}<0.05)$ (table 2).

\section{Immunohistochemical Stain for bcl-2 and bax}

bcl-2 showed a uniform staining of lymphocyte cytoplasm, whereas it was negative in malignant cells and normal gastric epithelial cells and in all 23 cases of the control group (GCLPS, fig. 3). bax was strongly expressed in malignant cell and normal lymphocyte cytoplasm (fig. 4). A positive reaction was seen in all 24 cases of GCLRS and 23 cases of the control group, GCLPS, with GCLRS showing $29.2 \%$ at $1+$ and $70.8 \%$ at $2+$ and GCLPS showing $30.4 \%$ at $1+$ and $69.6 \%$ at $2+$. None of the differences were statistically significant.

\section{Apoptosis Staining Using TUNEL}

Apoptosis was observed in all 24 cases of GCLRS and 23 cases of the control group (GCLPS), with 11 cases (45.8\%) showing $1+$ and 13 cases $(54.2 \%) 2+$ in GCLRS and 14 cases $(60.9 \%)$ showing $1+$ and 9 cases $(39.1 \%) 2+$ in GCLPS (table 2). Thus, slightly stronger expression was seen in GCLRS but this difference was not statisti- 
Fig. 3. Immunohistochemical staining for bcl-2. The malignant cells demonstrate negative immunoreactivity (black arrow) but infiltrating lymphocytes show positive immunoreactivity (white arrow). $\times 200$.

Fig. 4. Immunohistochemical staining for bax. The cytoplasm of the malignant cells shows brown granular positivity (arrow). $\times 200$.
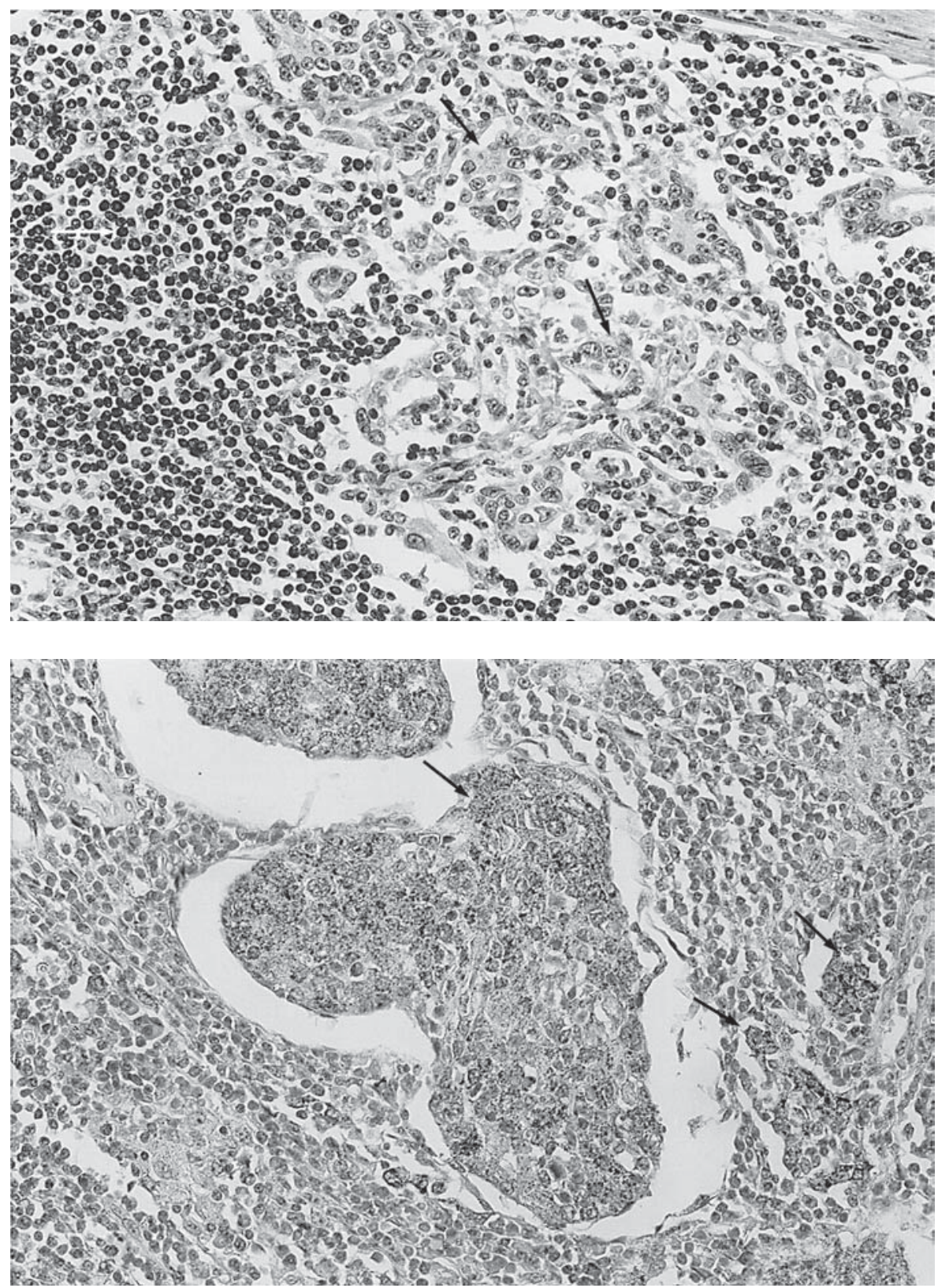

cally significant (fig. 5). No difference was seen in the percentage of cells that underwent apoptosis when 1,000 malignant cells per slide were evaluated $(p>0.05)$.

Although a significant correlation was present between the apoptotic index and bax expression $(\mathrm{p}<0.05)$, no significant difference was present between the degree of lymphocytic infiltration and the apoptotic index.

\section{Comparison of the Survival Period, Degree of}

Lymphocytic Infiltration, bax Expression, Apoptotic

Index, and EBER Expression in Stage III Gastric

Cancer

In the 43 cases followed ( 22 cases of GCLRS and 21 cases of GCLPS) the average survival period was 107 and 61 months, respectively. However, 50\% of GCLRS patients were stage I and most of these patients survived. Thus, to compare these patients in the same stage of gastric cancer with the control group, only stage III patients 
Fig. 5. TUNEL staining for apoptosis. In the malignant cells, occasional nuclear staining was noted (arrow). $\times 200$.

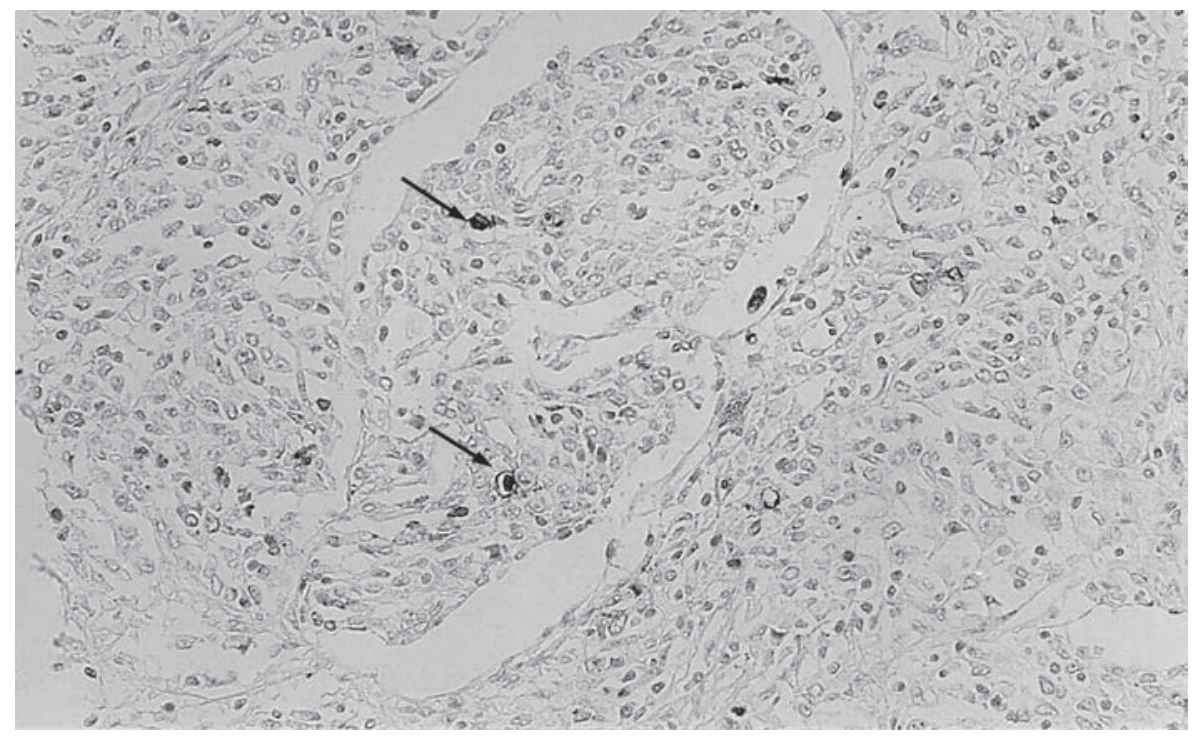

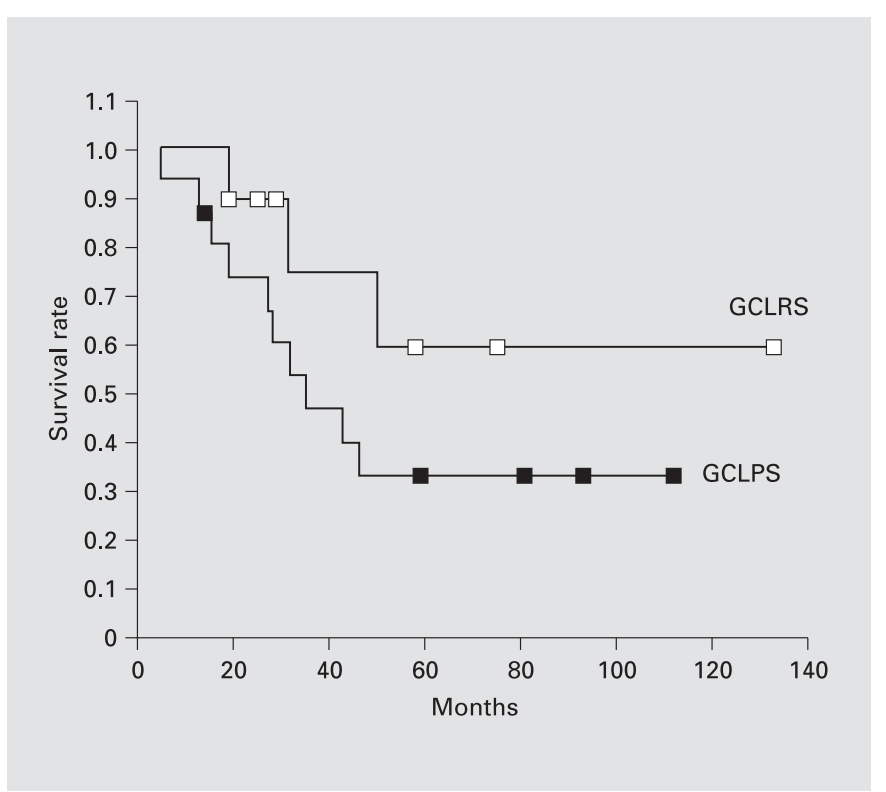

Fig. 6. The Kaplan-Meier survival curve of stage III cancers showed a better prognosis for patients with GCLRS compared to those with GCLPS with increasing length of follow-up.

were selected to compare and analyze the differences in the survival rate, EBER expression, bax expression, apoptosis and apoptotic index. Eleven cases of GCLRS and 17 cases of GCLPS were in stage III, whose average survival periods were 89 and 56 months, respectively. According to the Kaplan-Meier survival curve, no significant difference was present between the two groups. However, bet-
Table 3. Relationship between lymphocytic infiltration and EBER expression, bax, apoptosis and apoptotic index in stage III patients

\begin{tabular}{llccl}
\hline & & GCLRS $(\mathrm{n}=11)$ & $\operatorname{GCLPS}(\mathrm{n}=17)$ & $\mathrm{p}$ value \\
\hline EBV & negative & 1 & 17 & $<0.001$ \\
& positive & 10 & 0 & $\mathrm{NS}$ \\
\hline bax & $1+$ & 4 & 5 & \\
& $2+$ & 7 & 12 & $\mathrm{NS}$ \\
\hline Apoptosis & $1+$ & 6 & 11 & \\
& $2+$ & 5 & 6 & $\mathrm{NS}$ \\
\hline \multirow{2}{*}{ AI (mean } & $\pm \mathrm{SD})$ & $3.00 \pm 1.47$ & $2.52 \pm 1.47$ &
\end{tabular}

$\mathrm{AI}=$ Apoptotic index: $\%$ of apoptosis in 1,000 tumor cells.

ter prognosis was seen in patients with GCLRS with increasing follow-up period (fig. 6). EBER expression was significantly higher in the GCLRS group, but no significant difference was seen in bax expression, apoptosis and apoptotic index (table 3).

\section{Discussion}

After MacCarty and Mahle [20] reported that a better prognosis is seen in gastric carcinoma with lymphocytic infiltration within tumors, Watanabe et al. [2] described 
gastric carcinoma with lymphoid stroma characterized by abundant lymphocytic infiltration with no proliferation of connective tissue, differentiating it from usual gastric carcinoma. The incidence of this latter type of gastric carcinoma is very low at $1.1-4 \%$, but the emphasis is being placed on this carcinoma having characteristic histological findings and a good prognosis [2-7]. We defined those gastric carcinomas with no connective tissue within stroma and more than 30\% lymphocytic infiltration within tumors as GCLRS and found this type of gastric carcinoma in 24 cases, with an incidence of $1.47 \%$, in a total of 1,635 gastric carcinoma cases. This incidence was somewhat lower than that reported by Watanabe et al. [2] at 1.1-4\%, Matsunou et al. [6] at 3.7\%, and Chang et al. [7] at $4.6 \%$, probably because our criteria of GCLRS were more stringent (including only those cases which showed more than 30\% lymphocytic infiltration) and an accurate definition needs to be established in the future.

EBV infection not only participates in the development of lymphoproliferative disease and nasopharyngeal carcinoma but it is also related to the development of lesions similar to lymphoepithelioma in various organs [5-9]. Especially, the rate of positive expression of EBV in gastric carcinoma accompanied by lymphocytic infiltration was $82-90 \%$ [5-9], which is similar to the $79 \%$ found in the present study and higher than 67\% [7] according to a recent study done in Koreans. As shown in the results of the present study, the expression of EBV in GCLRS was significantly higher than in the control group (GCLPS). When EBV expression for EBER using in situ hybridization was observed in the present study, this expression was typically shown only in malignant cells but not in lymphocytes surrounding tumors or normal gastric mucosa, similar to the results reported by Shibata and Weiss [4] and Pittaluga et al. [8]. According to Harn et al. [9], malignant cells in gastric carcinoma infected with EBV all showed monoclonal EBV DNA, suggesting that this viral infection occurred before epithelial cells turned malignant. Shin et al. [11] claimed that malignant cells infected with EBV would undergo monoclonal growth by EBV infection participating in the early stage of cancer development, considering the findings that EBER was distributed evenly throughout gastric carcinoma nuclei and EBV is expressed in primary sites and metastatic sites. However, the exact role of EBV in the carcinogenesis of GCLRS has not been elucidated.

Kume et al. [12] reported that EBV-positive gastric adenocarcinomas had $b c l-2$ overexpression and a low rate of apoptosis. They also mentioned that a low apoptotic index was seen in EBV-positive GCLRS which was related to a good prognosis although the exact mechanism has not yet been elucidated. The expression of bcl-2 in gastric carcinoma varied according to each study. Lauwers et al. [21] mentioned that bcl-2 was expressed in most cases of the intestinal type of gastric adenocarcinoma but not in the diffuse type. On the other hand, Lee et al. [22] reported that bcl-2 was expressed strongly in diffuse type gastric adenocarcinoma. Moreover, Koshida et al. [23] reported that the apoptotic index was lower when bcl-2 was positive in gastric carcinoma, whereas it was not higher when bax was positive. On the other hand, there was a study reporting that the presence of $b c l-2$ expression was not related to the apoptotic index [8]. In EBV-associated malignancy such as Burkitt's lymphoma and nasopharyngeal carcinoma, it was reported that the modulation of apoptosis by LMP-1 or EBERs participates in carcinogenesis $[16,17]$; thus, the association between apoptosis and changes of apoptosis regulatory protein expression would be stronger in GCLRS having higher EBV expression. However, we could not find any study on apoptosis or apoptosis regulatory protein expression in GCLRS.

The frequency of apoptosis was examined in the present study in 24 cases of GCLRS and 23 cases of GCLPS using TUNEL, and immunohistochemical staining was done for bcl-2 and bax; bcl-2 showed good counterstaining with surrounding lymphocytic infiltration, but was negative in malignant cells in all cases. The finding of no bcl-2 expression in malignant cells was similar to the results of Lauwers et al. [21]. However, further studies are needed with more subjects since the results on $b c l-2$ expression vary in gastric carcinoma according to each author. bax is a protein that up-regulates apoptosis and was reported to be expressed in $92 \%$ of gastric carcinoma cases [18]. This protein was positive in almost all cases in the present study, with the only difference being the grades of expression. A significant relationship was seen between the grade of bax expression and apoptotic index $(\mathrm{p}<0.05)$, confirming the hypothesis that bax up-regulates apoptosis. However, no significant difference was seen between bax and apoptosis in the GCLRS and GCLPS groups, and no relationship was present with EBV expression $(p>0.05)$. However this conclusion must be treated with caution due to the potential variations in the interpretation of immunostaining. Further studies are in order on various factors known to be related to apoptosis, other than bcl-2 and bax.

Many studies on the prognosis of GCLRS have reported that the prognosis of this gastric carcinoma is bet- 
ter than that of usual gastric adenocarcinoma [5-9]. Matsunou et al. [6] reported that patients responded better to chemotherapeutic drugs since almost no connective tissue existed within the stroma in GCLRS. Although we could not compare the results of chemotherapy, a difference was seen in survival since at the time of surgery, $50 \%$ were in stage IA and B, $4.2 \%$ in stage II, and $45.8 \%$ in stage IIIA and B in the GCLRS group, whereas 33.1\% were in stage IA and B, $19.7 \%$ in stage II, and $38 \%$ in stage IIIA and B in the GCPLS group, which shows that more patients with GCLRS have lower stages. Considering the survival rates in the same clinical stage (stage III) according to lymphocytic infiltration, no significant difference was observed. However, the survival period differed with GCLRS patients achieving 89 months and GCLPS patients 56 months, and the survival curves were also distinct (fig. 6), suggesting that a significant difference could be expected as the follow-up period increases and cases of GCLRS were added.

\section{Conclusion}

The results indicate that GCLRS is a characteristic type of gastric carcinoma different from the intestinal type of gastric adenocarcinoma which is often related with $\mathrm{He}$ licobacter pylori infection or signet ring cell carcinoma. GCLRS is closely associated with EBV infection and shows the best prognosis among various types of gastric carcinoma. To understand the carcinogenesis of GCLRS and the role of EBV more studies need to investigate the effect of EBER on apoptosis in relation to double-stranded RNA-activated protein kinase in GCLRS, and the relationship between EBV LMP-1 and apoptosis.

\section{References}

1 Kim JP: Diagnosis, treatment and survival rate of 5 major cancers in Korea. J Korean Med Assoc 1995;38:157-164.

2 Watanabe H, Enjoji M, Imai T: Gastric carcinoma with lymphoid stroma. Cancer 1976;38: 232-243.

3 Cotran RS, Kumar V, Collins T: Pathologic Basis of Disease, ed 6. Philadelphia, Saunders, 1999, p 764.

4 Shibata D, Weiss LM: Epstein-Barr virus associated gastric adenocarcinoma. Am J Pathol 1992;140:769-774.

5 Nakamura S, Ueki T, Yao T, Ueyama T, Tsuneyoshi M: Epstein-Barr virus in gastric carcinoma with lymphoid stroma. Cancer 1994; 73:2239-2249.

6 Matsunou H, Konishi F, Hori H, Ikeda T, Sasaki K, Hirose Y, Yamamichi N: Characteristics of Epstein-Barr virus-associated gastric adenocarcinoma with lymphoid stroma in Japan. Cancer 1996;77:1988-2004.

7 Chang MS, Kim WH, Kim CW, Kim YI: Epstein-Barr virus-associated gastric carcinomas with lymphoid stroma. Histopathology 2000; 37:309-315.

8 Pittaluga S, Loke SL, So KC, Cheung KN, Ma L: Clonal Epstein-Barr virus in lymphoepithelioma-like carcinoma of the stomach: Demonstration of viral genome by in situ hybridization and southern blot analysis. Mod Pathol 1992;5:661-664.
9 Harn HJ, Chang JY, Wang MW: Epstein-Barr virus associated gastric adenocarcinoma in Taiwan. Hum Pathol 1995;26:267-271.

10 Tokunaga M, Land CE, Uemura Y, Tokudome T, Tanaka S, Sato E: Epstein-Barr virus in gastric carcinoma. Am J Pathol 1993;143:12501254.

11 Shin WS, Kang MW, Kang JH, Choi MK, Ahn BM, Kim JK, Sun HS, Min KW: Epstein-Barr virus associated gastric adenocarcinomas among Koreans. Am J Clin Pathol 1996;105: 174-181.

12 Kume T, Oshima K, Shinohara T, Takeo H, Yamashita Y, Shirakusa T, Kikuchi M: Low rate of apoptosis and overexpression of bcl-2 in Epstein-Barr virus-associated gastric carcinoma. Histopathology 1999;34:502-509.

13 Nanbo A, Takada K: The role of Epstein-Barr virus-encoded small RNAs (EBERs) in oncogenesis. Rev Med Virol 2002;12:321-326.

14 Zhang X, Hu L, Fadee B, Ernberg IT: Apoptosis modulation of Epstein-Barr virus-encoded latent membrane protein 1 in the epithelial cell line $\mathrm{HeLa}$ is stimulus-dependent. Virology 2002;304:330-341.

15 Koshida Y, Saegusa M, Okayasu I: Apoptosis, cell proliferation and expression of bcl-2 and bax in gastric carcinomas: Immunohistochemical and clinicopathological study. Br J Cancer 1997:75:367-373.
16 Yin XM, Oltvai ZN, Korsemeyer SJ: BH1 and $\mathrm{BH} 2$ domains of bcl-2 are required for inhibition of apoptosis and heterodimerization with bax. Nature 1994;369:321-323.

17 John FR, Clay MW, Brian VH: Apoptosis: Its significance in cancer and cancer therapy. Cancer 1994;73:2013-2026.

18 Bakhshi A, Jesen JP, Goldman P: Cloning the chromosomal breakpoint of $\mathrm{t}(14 ; 18)$ human lymphomas. Cell 1985;41:889-906.

19 Irvin DF, Jay SG, Donald EH, Robert H, Kennedy BJ, Gerald PM, et al: Manual for Staging of Cancer, ed 5. Philadelphia, Lippincott, 1997, pp 71-76.

20 MacCarty WC, Mahle AE: Relation of differentiation and lymphocytic infiltration to postoperative longevity in gastric carcinoma. J Lab Clin Med 1921;6:321-330.

21 Lauwers GY, Scott GV, Karpeh MS: Immunohistochemical evaluation of bcl-2 protein expression in gastric adenocarcinoma. Cancer 1995; 75:2209-2013.

22 Lee SS, Cho KJ, Hong SI, Myoung NK, Jang JJ: Nuclear overexpression of bcl-2 oncoprotein during the progression of human stomach cancer. J Korean Med Sci 1998;13:153-158.

23 Koshida Y, Saegusa M, Okayasu I: Apoptosis, cell proliferation and expression of bcl-2 and bax in gastric carcinomas: Immunohistochemical and clinicopathological study. Br J Cancer 1997; 75:367-373. 